\title{
The impact of climate on the abundance of Musca sorbens, the vector of trachoma
}

\author{
Anita Ramesh ${ }^{1 *}$, Julie Bristow ${ }^{2}$, Sari Kovats ${ }^{3}$, Steven W. Lindsay ${ }^{4}$, Dominic Haslam ${ }^{5}$ Elena Schmidt ${ }^{5}$ \\ and Clare Gilbert ${ }^{1}$
}

\begin{abstract}
Background: To assess the extent to which climate may affect the abundance of Musca sorbens, a putative vector of trachoma.

Data sources: Studies were identified by systematically searching online databases including CAB abstracts, Embase, Global Health, Medline, Web of Science and BIOS Online, references from key articles, and the websites of relevant international agencies.

Methods: A systematic literature review was conducted of field and laboratory studies that reported the impact of climate factors (e.g., temperature, humidity) on the synanthropic fly Musca sorbens. Data were systematically extracted and studies assessed for quality by two readers. Study results were reported narratively.

Results: A total of 16 studies met the inclusion criteria but only three evaluated associations between climatic/ abiotic factors and $M$. sorbens. Limited evidence indicates that $M$. sorbens abundance has an optimal temperature and humidity range. Thirteen studies reported seasonal patterns but no consistent pattern was found between season and the abundance of $M$. sorbens.
\end{abstract}

Conclusions: The evidence base regarding the effect of climatic factors on M. sorbens is limited, so it is difficult to construct a biological model driven by climate for this species. A multivariate statistical approach based on the climate of sites where M. sorbens is found may better capture its complex relationship with climatic factors as well as aid in mapping the global range of $M$. sorbens.

Keywords: Musca sorbens, Diptera, Flies, Climate, Temperature, Rainfall, Humidity, Trachoma, Transmission

\section{Background}

Trachoma, caused by the bacterium Chlamydia trachomatis, is the world's leading infectious cause of blindness. It is thought to be endemic in 51 countries, primarily in sub-Saharan Africa (Fig. 1) [1]. It is estimated that 232 million people living in trachomaendemic areas are at risk of the condition; of the over 1.8 million who are visually impaired due to trichiasis (in-turned eyelashes) and its complications, over 500,000 people are irreversibly blind. International efforts are underway to map affected populations and scale-up

\footnotetext{
* Correspondence: anita.ramesh@lshtm.ac.uk

${ }^{1}$ Department of Clinical Research, Faculty of Infectious and Tropical Diseases (ITD), London School of Hygiene \& Tropical Medicine (LSHTM), Keppel Street, London, UK

Full list of author information is available at the end of the article
}

interventions to eliminate blinding trachoma as a public health problem [2-4].

Chlamydia trachomatis can be transmitted directly from person-to-person via contact with infected ocular or nasal secretions, or indirectly from fomites via contact with items such as washcloths and bedding. However, eye-seeking flies - such as the 'bazaar fly' Musca sorbens (Diptera: Muscidae) in Africa and Asia, the 'bush fly' M. vetustissima in Australia, and eye gnats Hippelates spp. and Liohippelates spp. in Latin America have also been implicated in trachoma transmission to varying degrees [5-9]. Female flies feed on human ocular and nasal secretions in order to obtain the nutrients necessary for egg production, so are thought to play a substantial role in fly-related mechanical transmission of trachoma [10]. However, the relative importance of flies in trachoma transmission is likely to vary by geographic 


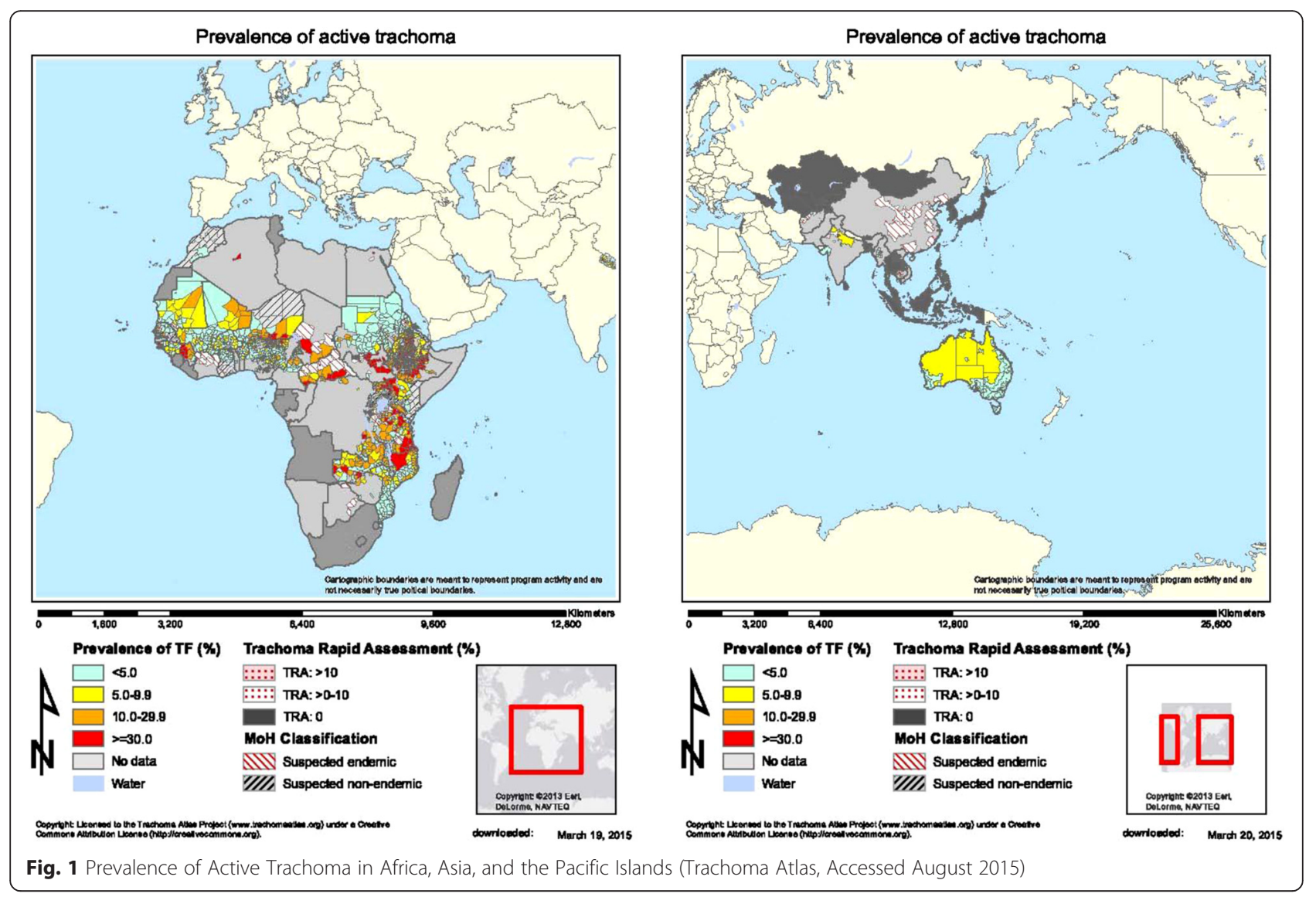

location, socio-economic development levels, and time of year. Evidence for a role of $M$. sorbens in the mechanical transmission of trachoma comes from both observational studies in which high fly densities on or around human faces have been associated with trachoma as well as intervention studies in which reductions in $M$. sorbens densities have been associated with a lower incidence of C. trachomatis infection $[5,7]$. For example, in The Gambia, a randomised controlled trial demonstrated that, insecticide spraying or the provision of pit latrines was associated with a lower incidence of trachoma compared to no intervention [7]. In Tanzania, another trial of insecticide spraying after mass distribution of antibiotics led to a $39 \%$ lower prevalence of trachoma after six months spraying; although this was of borderline significance, after 12 months there was no difference in trachoma prevalence despite consistent reductions in $M$. sorbens abundance [11]. The relative importance of $M$. sorbens as a trachoma vector is likely to vary between geographic locations and change over time.

The geographical distribution of $M$. sorbens overlaps with areas of active transmission of trachoma, as there are areas where $M$. sorbens exists which are not endemic for trachoma (Fig. 1) [12]. A recent systematic literature review of climatic effects on the prevalence, distribution and severity of trachoma concluded that the current distribution of trachoma is associated with higher temperatures, and the prevalence is lower at higher altitudes [13]. This is consistent with what one would expect if flies facilitate transmission [9].

As has been shown for other insect species, climate affects several stages in the life-cycle of $M$. sorbens (Fig. 2) [14-16]. The vectorial capacity of $M$. sorbens is dependent on the survival of adult flies, the proportion of flies carrying infectious $C$. trachomatis, the number of flies that land on a face, and the proportion of these flyhuman contacts that leads to infection. The development, survival and activity of flies is likely to be associated with ambient temperature and humidity. There is also an optimum range of temperature and humidity for the fly, above and below which the fly will fail to thrive (Fig. 3) [17-20]. Climate may also influence the abundance of $M$. sorbens through effects on environmental mediators, such as access to faeces for egg production, breeding, and maturation. Adult females preferentially oviposit on human faeces close to the ground, and the number and size of emerging offspring are greater for human than herbivore faeces, suggesting that human faeces provides the optimal conditions for fly development $[6,8,21,22]$. High temperatures combined with 


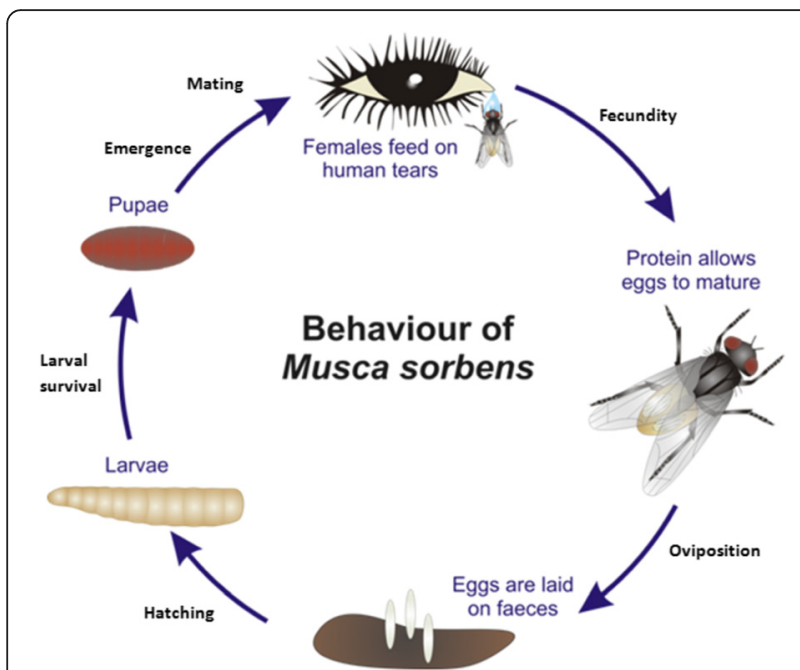

Fig. 2 The Life Cycle Of Musca sorbens, the Eye-Seeking Fly Implicated in Trachoma Transmission. (Rothamsted Research Visual Communications Unit, Reproduced with Permission)

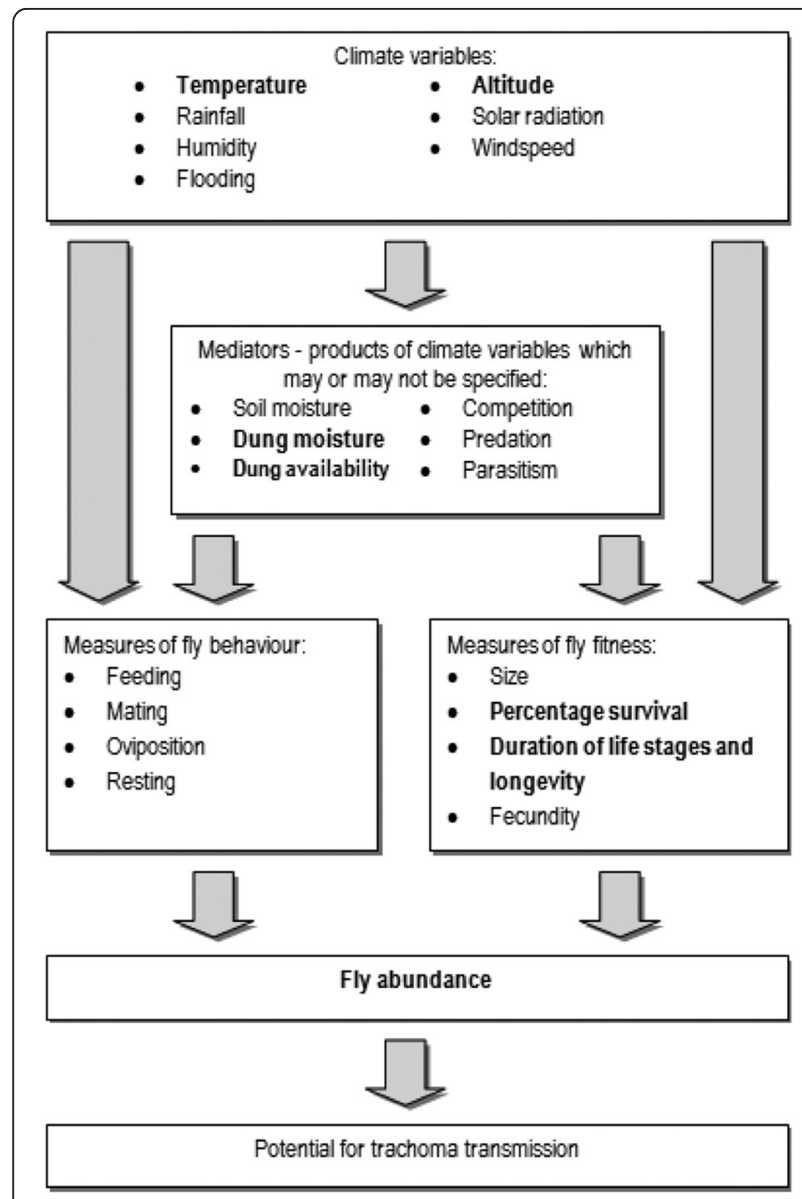

Fig. 3 Potential Effects of Climatic Factors and Mediators on Fly Abundance, Fitness, and Trachoma Transmission (Factors Found to be Associated with M. Sorbens from Retrieved Papers Marked in Bold) low humidity could dry out faeces or lead to crust formation on faeces, with the potential to limit adult emergence [23]. Climatic conditions that favour the growth of dung beetle populations, such as warm and wet weather, could lead to a reduction in $M$. sorbens; since dung beetles can remove dung from the soil surface within several hours [24].

The purpose of this study was to review the availability and quality of the evidence for the impact of climate factors on $M$. sorbens abundance, and to identify gaps in the literature on this under-researched vector.

\section{Methods}

A systematic literature review was conducted to evaluate the evidence for an association between climatic factors and the abundance of $M$. sorbens. Additional file 1: Table S1 describes the search terms, key words, and MESH headings used. The following outcomes in relation to $M$. sorbens were included: 1) abundance of adult flies; 2) fitness (size, life span/longevity, basic reproductive number and parity); 3) adult fly behaviour (e.g., daily activity times, feeding, mating, and oviposition); and 4) life cycle (i.e., development and maturation of eggs, pupae, and larvae). Climate-related exposure terms included: meteorological factors (temperature, rainfall, humidity, sunlight), weather events (e.g., drought, floods), and the related abiotic exposures for laboratory based studies (e.g., temperature, humidity, radiation). As $M$. sorbens is largely exophilic, fly count data from catches made outdoors are reported [25].

\section{Inclusion criteria}

Peer reviewed journal articles published between 1 January 1950 and 1 August 2013; field studies (observational, crosssectional, intervention or spatial) regarding the effect of climate factors on the abundance of $M$. sorbens; laboratory studies reporting an association between temperature, humidity, or other abiotic factors and $M$. sorbens outcomes, and studies describing seasonal patterns of fly counts.

\section{Exclusion criteria}

Review articles and conference abstracts.

There were no restrictions on geographic location of study, or language of journal publication.

Structured searches were conducted in the following standardized electronic databases: CAB, abstracts, Embase, Global Health, Medline, Web of Science and BIOS Online. Reference lists of selected articles were examined to find any other relevant papers. Limited searches were conducted among the following expert sources: World Health Organization (WHO) Special Programme for Research and Training in Tropical Diseases (TDR): http://www.who.int/tdr/en/; United Nations Children's Fund: http://www.unicef.org/; The 
Carter Center: http://www.cartercenter.org/; The International Trachoma Initiative (ITI): http://trachoma.org/; Tropical Diseases Bulletin from 1900 to 2010.

\section{Data extraction and analysis}

The review was conducted by two readers (JB and AR). An initial search was conducted of each database for all fly species implicated in trachoma transmission (see Additional file 1: Table S1). Titles were scanned to ensure that articles related to at least some aspect of the study question. Titles and abstracts were then reviewed to exclude papers that did not meet the inclusion criteria. Full texts of papers provisionally included were then obtained and inclusion criteria checked by the second reader. Data were then extracted using a standard format to collect data on study setting, design and target population, the nature of the study, climate factors and how they were measured, fly outcomes and how they were measured, and whether there were any statistical tests of association.

\section{Quality assessment}

A modified version of a quality assessment tool developed for quantitative studies was used to assess the quality of each study [26]. Each study was assessed as providing strong, moderate or weak evidence depending on methods of sampling, duration of sample, sample size, minimization of bias, control of confounding, and exposure assessment. Studies that met the criteria were assessed as having high, moderate, or poor quality. Studies graded to be of poor quality included studies where only descriptive data on fly abundance were presented.

It was anticipated that heterogeneity in study designs and exposure measurements would preclude a metaanalysis; thus, findings were compiled and reported using a narrative synthesis.

\section{Results}

From 11,130 that were initially recovered, a total of 16 studies met the criteria for our review (Fig. 4). Of the original 11,130 studies initially recovered, 10,833 were removed as duplicates or completely non-topical. Of remaining 297 abstracts screened for full review, 252 studies were excluded because they lacked data on flies, climate variables, or both, and one article was not based on primary data.

The 16 studies included in this review were heterogeneous with respect to outcome measures and methods used. Most studies were from previous decades, and were undertaken in a wide range of locations around the world. Most were field studies, and only one laboratorybased study was identified. Table 1 describes the two studies that were assessed to be of highest quality, Table 2 details the one study assessed to be of moderate quality, while Table 3 provides information on the thirteen studies deemed to be of low quality while still

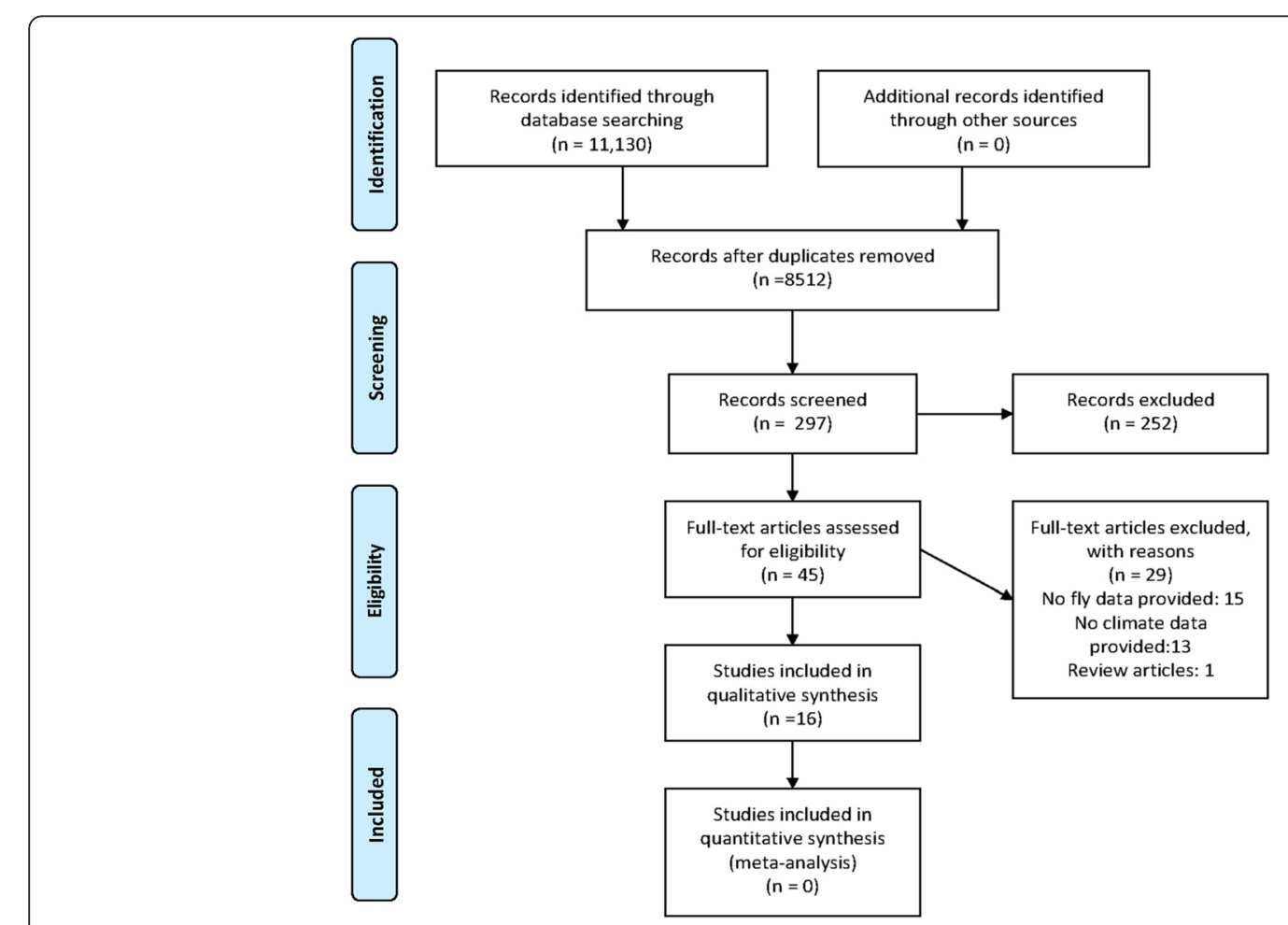

Fig. 4 PRISMA Process of Paper Selection 
Table 1 Effects of Climatic Factors on the Abundance of M. sorbens, High Quality Studies

Type, and Location Measure(s)

Fly Outcomes Methods

Results (Prevalence/Odds Ratio, $95 \%$

Confidence Interval (CI)) Where noted

$\mathrm{Cl}=$ Credibility Interval)

Authors: Toyama \& Tempera-ture: $12 \mathrm{~mm}$ below $\quad$ Adult: $\quad$ Method: Flies trapped for $5 \mathrm{~min}$ using

|keda, 1981 [23] surface of dung pat abundance cone traps baited with fresh cow dung

pats; Frequency: Bi-weekly for 11 months

Adult M. sorbens is significantly more

abundant in summer than winte

$(p<0.001)$.

Type: Field study Season: Summer (May - October)

and winter (November - April);

then weekly for an additional 14 months.

Egg and larval Method: Dung pat infestation rates: 20

Location: Dairy year unstated USA abundance randomly selected dung pats were examined for eggs and 20 for larvae. Frequency: Weekly between 10:00-20:00 h, for 15 months.

Larval abundance

Method: Dung pat larval examinations: 4 dung pats more than 3 days old were collected and examined. Frequency Monthly for 13 months.

Authors: Taye et al., t, November 2003 and February 2007 [9]

Type: Field study Altitude: $<2000 \mathrm{~m}$

2200-2500 m

$>3000 \mathrm{~m}$

Location: 12

villages in Gurage

Season: May, August, November 2003 and February 2004

zone, Ethiopia.
Adult

abundance

Method: Human landing catches on young children: counts of fly-eye contacts over

10 min outdoors, a short break, then

10 min indoors between 8:30 and 13:00.

Frequency: Bi-weekly for 11 months then

weekly for an additional 14 months.

Adult

abundance

Method: Modified WHO-exit traps baited with $M$. sorbens numbers declined with beef 10 traps over $25 \mathrm{~h}, 1.5 \mathrm{~m}$ above ground increasing altitude - of the 3465 in playground, plantation, pits, shade, and M. sorbens trapped, $55.6 \%$ and $44.3 \%$ indoors Frequency: Weekly between the hours of

Significantly more dung pats with eggs and larvae found in summer $(p<0.001)$. Dung pat temperatures were higher than ambient temperatures only in summer (ambient 29.16 \pm 0.80 C, dung pat $33.36 \pm 1.14 \mathrm{C} ; p<0.001)$. Dung pats developed thick, hard crusts during summer, but were thin and softened each night from moisture during winter. There were significantly more flies in unsoftened than softened pats $(p<0.001)$, and higher rates of summer (14\%, ns).

Almost all eye-seeking $M$. sorbens were captured outdoors at low and at medium altitudes [99.3 \%; $95 \%$ confidence interval (CI) $99.1 \%-99.5 \%$; $p<0.001]$.

Direct effects: Medium altitude is significantly associated with greater abundance of adult $M$. sorbens. temperature) is significantly associated with greater abundance fe stages of Musca sorbens.

Indirect effects: Crusts on dung pat at higher summer temperatures parasites which predate developing $M$ sorbens. Greater abundance of $M$ sorbens in summer may therefore reflect lower predation i.e, an indirect effect of temperature.

were collected in the low- and

mid-altitude villages, respectively, but only $0.1 \%(p=0.001)$ came from villages at higher altitudes. 
Table 2 Effects of Climatic Factors on the Abundance of M. sorbens, Moderate Quality Studies (from Hafez and Attia, 1958 [22])

\begin{tabular}{|c|c|c|c|c|c|}
\hline $\begin{array}{l}\text { Authors, Study Type, } \\
\text { and Location }\end{array}$ & $\begin{array}{l}\text { Climate Exposure(s), } \\
\text { Measure(s) }\end{array}$ & Fly Outcomes & Methods & $\begin{array}{l}\text { Results (Prevalence / Odds Ratio, } \\
95 \% \text { Confidence Interval (CI)) } \\
\text { Where noted Cl=Credibility Interval) }\end{array}$ & Conclusions \\
\hline $\begin{array}{l}\text { Authors: Hafez and } \\
\text { Attia, } 1958 \text { [22] }\end{array}$ & \multirow[t]{6}{*}{ Temperature } & $\begin{array}{l}\text { Egg incubation } \\
\text { period }\end{array}$ & $\begin{array}{l}\text { Humidity maintained at } 100 \% \text { RH. } \\
\text { Between } 53 \text { and } 60 \text { eggs were studied }\end{array}$ & $\begin{array}{l}\text { Eggs failed to hatch at } 5.5^{\circ} \mathrm{C} \text { or } \\
43^{\circ} \mathrm{C} \text {. Eggs took an average of }\end{array}$ & \multirow{3}{*}{$\begin{array}{l}\text { Within temperature limits that permitted } \\
\text { hatching, incubation period was inversely } \\
\text { proportional to temperature. Optimal } \\
\text { hatching occurred at } 28 \mathrm{C} \text {. No statistical } \\
\text { measures. }\end{array}$} \\
\hline Type: Lab study & & & $\begin{array}{l}\text { at } 5.5^{\circ} \mathrm{C}, 16^{\circ} \mathrm{C}, 24^{\circ} \mathrm{C}, 28{ }^{\circ} \mathrm{C}, 32{ }^{\circ} \mathrm{C}, 36^{\circ} \mathrm{C} \text {, } \\
40{ }^{\circ} \mathrm{C} \text { and } 43{ }^{\circ} \mathrm{C} \text {. }\end{array}$ & $\begin{array}{l}5.28 \mathrm{~h} \text { to hatch at } 40{ }^{\circ} \mathrm{C} \text { and } 25.3 \mathrm{~h} \\
\text { at } 16^{\circ} \mathrm{C} \text {. }\end{array}$ & \\
\hline \multirow[t]{4}{*}{$\begin{array}{l}\text { Location: Taliba, } \\
\text { Egypt. }\end{array}$} & & $\begin{array}{l}\text { Egg hatching } \\
\text { percentage }\end{array}$ & $\begin{array}{l}\text { Eggs were fertile and secured from } \\
\text { copulated females. }\end{array}$ & $\begin{array}{l}\text { At } 16{ }^{\circ} \mathrm{C} 95 \% \text { of eggs hatched. At } \\
28^{\circ} \mathrm{C} 100 \% \text { of eggs hatched. At } \\
36{ }^{\circ} \mathrm{C} 90 \% \text { of eggs At } 40{ }^{\circ} \mathrm{C} 65 \% \\
\text { of eggs hatched. }\end{array}$ & \\
\hline & & $\begin{array}{l}\text { Larvae Duration of } \\
\text { larval period }\end{array}$ & $\begin{array}{l}\text { Larvae were reared on } 15 \mathrm{~g} \text { milk/bran } \\
\text { larval diet, } 10 \text { eggs in each } 10 \mathrm{cc} \text { tube. } \\
\text { They were then emptied onto a drier } \\
\text { pupation medium. }\end{array}$ & $\begin{array}{l}\text { At } 16^{\circ} \mathrm{C} \text { the mean duration of the } \\
\text { larval period was } 268.95 \pm 0.94 \mathrm{~h} \text {. } \\
\text { At } 40 \mathrm{C} \text { it was } 73.05 \pm 0.71 \mathrm{~h} \text {. }\end{array}$ & $\begin{array}{l}\text { Larval period duration was inversely } \\
\text { proportional to temperature. Failure } \\
\text { to provide drier pupation medium } \\
\text { prolonged the larval period duration by } \\
\text { several days. No statistical measures. }\end{array}$ \\
\hline & & $\begin{array}{l}\text { Pupae Duration of } \\
\text { pupal period }\end{array}$ & $\begin{array}{l}\text { Humidity maintained at } 70 \% \mathrm{RH} .42-58 \\
\text { pupae were studied at temperatures of } \\
16^{\circ} \mathrm{C}, 24^{\circ} \mathrm{C}, 28^{\circ} \mathrm{C}, 32^{\circ} \mathrm{C}, 36^{\circ} \mathrm{C} \text { and } 40^{\circ} \mathrm{C} \text {. }\end{array}$ & $\begin{array}{l}\text { At } 16^{\circ} \mathrm{C} \text { the mean duration of the } \\
\text { pupal period was } 14.9 \pm 0.2 \mathrm{~h} \text {. At } \\
36^{\circ} \mathrm{C} \text { it was } 3.86 \pm 0.02 \mathrm{~h} \text {. }\end{array}$ & $\begin{array}{l}\text { Pupal period duration was inversely } \\
\text { proportional to temperature. }\end{array}$ \\
\hline & & $\begin{array}{l}\text { Adult Percentage } \\
\text { adult emergence }\end{array}$ & $\begin{array}{l}\text { Humidity maintained at } 70 \% \text { RH. } 90-115 \\
\text { pupae were studied at temperatures of } \\
16^{\circ} \mathrm{C}, 24^{\circ} \mathrm{C}, 28^{\circ} \mathrm{C}, 32^{\circ} \mathrm{C}, 36^{\circ} \mathrm{C} \text { and } 40^{\circ} \mathrm{C} \text {. }\end{array}$ & $\begin{array}{l}66.6 \% \text { of adults emerged at } 16^{\circ} \mathrm{C} \text {. } \\
94.7 \% \text { emerged at } 24^{\circ} \mathrm{C} \text { and } \\
28^{\circ} \mathrm{C} .90 \% \text { emerged at } 32^{\circ} \mathrm{C} \text {. None } \\
\text { emerged at } 40^{\circ} \mathrm{C} \text {. }\end{array}$ & $\begin{array}{l}\text { Maximum emergence occurred } \\
\text { between } 24 \mathrm{C} \text { and } 28 \mathrm{C} .40 \mathrm{C} \\
\text { temperatures prevent emergence. } \\
\text { No statistical measures. }\end{array}$ \\
\hline $\begin{array}{l}\text { Authors: Hafez and } \\
\text { Attia, } 1958 \text { [22] }\end{array}$ & \multirow[t]{4}{*}{ Relative Humidity } & $\begin{array}{l}\text { Egg incubation } \\
\text { period }\end{array}$ & $\begin{array}{l}\text { Temperature maintained at } 31-32^{\circ} \mathrm{C} \text {. No } \\
\text { information given on numbers used. }\end{array}$ & $\begin{array}{l}\text { At } 100 \% \text { humidity eggs took } 6.3-6.6 \mathrm{~h} \\
\text { to hatch. At } 95 \% \text { humidity eggs took }\end{array}$ & \multirow{4}{*}{$\begin{array}{l}\text { Eggs did not hatch at humidities below } \\
85 \% \text {, and took longer to hatch at lower } \\
\text { humidities. Fewer eggs hatched at lower } \\
\text { humidities. Low humidities damaged } \\
\text { eggs through dehydration, causing } \\
\text { shrinkage. No statistical measures. }\end{array}$} \\
\hline Type: Lab study & & & & $\begin{array}{l}\text { eggs took } 7.1-6.6 \text { h to hatch. At } 85 \% \\
\text { humidity no eggs hatched. }\end{array}$ & \\
\hline \multirow[t]{2}{*}{$\begin{array}{l}\text { Location: Taliba, } \\
\text { Egypt. }\end{array}$} & & $\begin{array}{l}\text { Egg hatching } \\
\text { percentage }\end{array}$ & $\begin{array}{l}\text { Temperature maintained at } 31-32^{\circ} \mathrm{C} \text {. No } \\
\text { information given on numbers used. }\end{array}$ & $\begin{array}{l}\text { At } 100 \% \text { humidity } 100 \% \text { of eggs } \\
\text { hatched. At } 90 \% \text { humidity } 58 \% \text { of } \\
\text { eggs hatched. At } 85 \% \text { humidity no } \\
\text { eggs hatched. }\end{array}$ & \\
\hline & & Egg structure & $\begin{array}{l}\text { Fertile eggs laid within } 15 \text { min of the start } \\
\text { of the experiment were placed in a desiccator. }\end{array}$ & $\begin{array}{l}\text { Egg length/width: When RH was } \\
\text { increased from } 0 \text { to } 100 \% \text { length } \\
\text { increased from } 1.369 \mathrm{~mm} \text { to } 1.465 \mathrm{~mm} \\
\text { (average elongation } 7 \% \text { ) and width } \\
\text { increased from } 0.298 \mathrm{~mm} \text { to } 0.312 \mathrm{~mm} \\
\text { (average increase in width of } 4.7 \% \text { ). }\end{array}$ & \\
\hline
\end{tabular}

Pupae Duration of Humidity maintained at $70 \%$ RH. 42-58

Temperature maintained at $31-32{ }^{\circ} \mathrm{C}$. No information given on numbers used.

\section{Attia, 1958 [22]}

period

Egg hatching

Temperature maintained at $31-32^{\circ} \mathrm{C}$. No

Fertile eggs laid within 15 min of the start

(average increase in width of $4.7 \%$ ). 
Table 2 Effects of Climatic Factors on the Abundance of M. sorbens, Moderate Quality Studies (from Hafez and Attia, 1958 [22]) (Continued)

Pupae Duration of Temperatures of $28^{\circ}$ and $36^{\circ} \%$.

pupal period

No information given on numbers used.
Egg weight (water loss): When exposed

to $30 \% \mathrm{RH}$, eggs lost $30 \%$ of their

weight in first $60 \mathrm{~min}(20 \%$ in first

$30 \mathrm{~min}, 10 \%$ in last $30 \mathrm{~min})$. Water loss

decreased with increasing exposure to

low RH.

The pupal phase lasted 106-118 $\mathrm{h}$ at

$28^{\circ} \mathrm{C}$ regardless of humidity, and

between 90 and $93 \mathrm{~h}$ at $36^{\circ} \mathrm{C}$

Relative humidity has no significant effect on duration of the pupal phase. 
Table 3 The Effect of Climatic Factors and Seasonality on M. sorbens Abundance. Low Quality Studies

\begin{tabular}{|c|c|c|c|c|}
\hline $\begin{array}{l}\text { Reference and } \\
\text { Study Location }\end{array}$ & $\begin{array}{l}\text { Fly } \\
\text { Outcome }\end{array}$ & Methods & Results & Climatic or Seasonal Effects \\
\hline $\begin{array}{l}\text { Author(s): } \\
\text { Ponghis (1957) } \\
\text { [36] }\end{array}$ & $\begin{array}{l}\text { Adult } \\
\text { abundance }\end{array}$ & Collection type: Traps (type unstated) & \multirow{2}{*}{$\begin{array}{l}\text { Mean monthly catches were highest } \\
\text { in June and October, when } M \text {. sorbens } \\
\text { comprised } 1 \% \text { and } 30 \% \text {, respectively, } \\
\text { in relation to } M \text { domestica. From } \\
\text { databases: June: average max temp } \\
24{ }^{\circ} \mathrm{C} \text {, Oct } 25^{\circ} \mathrm{C} \text {; June av ppt } 1 \mathrm{~mm} \text {; }\end{array}$} & \multirow{2}{*}{$\begin{array}{l}\text { M. sorbens abundance was greatest in } \\
\text { spring and autumn (i.e., just before } \\
\text { and after the hottest, driest months). } \\
\text { Heat and dryness had an } \\
\text { unfavourable effect on fly } \\
\text { multiplication. No statistical measures. }\end{array}$} \\
\hline $\begin{array}{l}\text { Location: } 2 \\
\text { villages, } \\
\text { Southern }\end{array}$ & & $\begin{array}{l}\text { Frequency: } 1 \text { x / week, June - } \\
\text { December (1956) }\end{array}$ & & \\
\hline
\end{tabular}

Southern

Morocco

Other details: Traps baited with human faeces, fruit, or vegetables. Precautions taken to eliminate effects of secondary factors (e.g., timing of trap deployment).

Author(s):
Miranpuri and
Lahkar (1980)
[35]
Location: Cattle
sheds, Assam,
India

\section{Adult \\ Collection type: Fly nets} abundance

Frequency: $4 \mathrm{x} /$ month, $2 \mathrm{~h}$ twice per day (8-10 am; 13-15 pm); 19745 (exact period unstated).

Author(s): Amin et al. (1998) [30]

Location: Al Amargh, Cairo (outskirts),

Egypt.

Author(s): Khan et al. (1965) [32]

Location: India

(National Study)

Author(s):
Rechav (1989)
[37]

Location: Cattle sheds, Transvaal, South Africa

Author(s):
Sukhova (1963)
[38]

Location: South, Southwest and Northern

Turkey.

Author(s):
Tawfik (1969)
[39] N

Location: Cairo (outskirts), Egypt.
Adult abundance

\section{Collection type: Cone traps}

Frequency: 1 x / month, December 1994 - November 1995

Other details: Traps baited with meat, fish, or liver "near fly breeding sites".

Adult abundance

Collection type: Method not stated

Frequency: 3 x / day, 30 min each (duration not provided)

Other details: Altitude $(m)$ per location: 11 m (Bombay) - >2000 m (Simla).

\section{Adult Collection type: Hand net} abundance

Frequency: $1 \times$ month, collected between 11 am - 12 pm each day over 14 months (years unstated).

Other details: $10 \mathrm{ml}$ of fresh blood was poured on 12 cows.

Adult Collection type: Fly traps abundance

Frequency: Time / duration not provided; day and night trapping reported.

Adult Collection type: Wire mesh cone abundance traps

Frequency: 2 x / month, hourly

Other details: Traps baited with rotten fish. catches on two consecutive days
M. sorbens numbers appear to peak between July - October, with the highest peaks in August (end of hotwet season) and September (start of dry-hot season). From databases: April: Av max temp: $28^{\circ} \mathrm{C}$ and av ppt $3 \mathrm{~mm}$. June Av max temp: $35^{\circ} \mathrm{C}$ and av ppt $0 \mathrm{~mm}$ August Av max temp $31{ }^{\circ} \mathrm{C}$, av ppt: $462 \mathrm{~mm}$.

Relative distribution of fly species by season. Mean monthly catches were highest in April, February and August, and lowest in November, September and July. From databases: April: Av max temp: $28^{\circ} \mathrm{C}$ and av ppt $3 \mathrm{~mm}$. June Av max temp: $35^{\circ} \mathrm{C}$ and av ppt $0 \mathrm{~mm}$

M. sorbens is "essentially a fly of the plains, being entirely absent at higher elevations". In Chandigargh (387.5 m), for instance, it comprised over $78 \%$ of flies caught whereas in Simla (2202 m) none were caught.

M. sorbens accounted for $1.6 \%$ of all flies caught.

M. sorbens numbers peak in August/ September. None were caught in many months outside this season. From databases: August av max temp: $22{ }^{\circ} \mathrm{C}$ and av ppt $5 \mathrm{~mm}$; September av max temp: $25^{\circ} \mathrm{C}$ and av ppt $20 \mathrm{~mm}$

$M$ sorbens displayed two seasonal peaks (summer, autumn). In the north, $M$ sorbens peaked in summer only. From databases: Hottest summer months June to August: Av max temp: $35-36{ }^{\circ} \mathrm{C}$ and av ppt 33-38 mm.

Daily temperature: $M$. sorbens numbers were highest on days with average daily temperatures of $26-26.7^{\circ} \mathrm{C}$; in summer, $M$. sorbens numbers peaked between 6 and $7 \mathrm{am}$, and 5 and $7 \mathrm{pm}$ whereas in winter, $M$ sorbens numbers peaked at 8 am then rose with temperature to peak at $12 \mathrm{pm}$.

Season/temperature/humidity: $M$ sorbens numbers were highest in spring (April), corresponding to mean
M. sorbens abundance was greatest towards the end of the hot, wet season and the beginning of the dryhot season. No statistical measures.

M. sorbens accounted for $8.5 \%$ of all flies caught throughout the year. $M$ sorbens demonstrated two seasonal peaks, in April and June, when it was the second or third most populous species caught. No statistical measures.

M. sorbens was not found at high altitude (>2000 m), a proxy for low temperature. No statistical measures.

M. sorbens abundance was greatest in late winter / early spring, but were not abundant at any time in this study. No statistical measures.

No numbers only figures provided. No statistical measures.

M. sorbens abundance was greatest at higher temperatures i.e., 22-23C. No statistical measures. 
Table 3 The Effect of Climatic Factors and Seasonality on M. sorbens Abundance. Low Quality Studies (Continued)

\begin{tabular}{|c|c|c|c|c|}
\hline & & & $\begin{array}{l}\text { temperature of } 22.9^{\circ} \mathrm{C} \text { and } \mathrm{RH} 38 \% \text {; } \\
\text { second peak in autumn (October) with } \\
\text { temperature } 22.8^{\circ} \mathrm{C} \text { and } \mathrm{RH} \text { of } 56 \% \text {. }\end{array}$ & \\
\hline $\begin{array}{l}\text { Author(s): Hafez } \\
\text { and Attia (1958) } \\
\text { [21] }\end{array}$ & \multirow[t]{2}{*}{$\begin{array}{l}\text { Adult } \\
\text { abundance }\end{array}$} & $\begin{array}{l}\text { Collection type: method not reported } \\
\text { Frequency: } 1 \text { x / week, entire year } \\
\text { (1957) }\end{array}$ & \multirow[t]{2}{*}{$\begin{array}{l}\text { Mean monthly catches were highest } \\
\text { in April, February and August, and } \\
\text { lowest in November, September and } \\
\text { July. }\end{array}$} & \multirow[t]{2}{*}{$\begin{array}{l}\text { M. sorbens abundance was greatest in } \\
\text { spring and summer and were lowest } \\
\text { in winter. No statistical measures. }\end{array}$} \\
\hline $\begin{array}{l}\text { Location: Cairo } \\
\text { (outskirts), } \\
\text { Egypt. }\end{array}$ & & $\begin{array}{l}\text { Other details: Flies 'attracted to } \\
\text { children's' eyes' were collected. }\end{array}$ & & \\
\hline \multirow{3}{*}{$\begin{array}{l}\text { Author(s): Koe } \\
\text { (1975) } \\
\text { Location: } 1 \\
\text { county, Central } \\
\text { China }\end{array}$} & \multirow[t]{3}{*}{$\begin{array}{l}\text { Adult } \\
\text { abundance }\end{array}$} & $\begin{array}{l}\text { Collection type: Traps (method not } \\
\text { reported) }\end{array}$ & \multirow{3}{*}{$\begin{array}{l}\text { M. sorbens numbers appear to peak in } \\
\text { August, and are lowest in April. It was } \\
\text { the fourth most populous species } \\
\text { caught. }\end{array}$} & \multirow[t]{3}{*}{$\begin{array}{l}\text { M. sorbens abundance was greatest in } \\
\text { late summer. No statistical measures. }\end{array}$} \\
\hline & & $\begin{array}{l}\text { Frequency: } 1 \text { x / month, April - } \\
\text { November } 1963\end{array}$ & & \\
\hline & & $\begin{array}{l}\text { Other details: Fermented bran and } \\
\text { sugar bait-trap method. }\end{array}$ & & \\
\hline \multirow{2}{*}{$\begin{array}{l}\text { Author(s): Wang } \\
\text { at al. (2000) [40] }\end{array}$} & \multirow{3}{*}{$\begin{array}{l}\text { Adult } \\
\text { abundance }\end{array}$} & Collection type: Cylindrical traps & \multirow{2}{*}{$\begin{array}{l}\text { M. sorbens was the first most populous } \\
\text { species caught in } 1998 \text { and the } \\
\text { second in } 1999 .\end{array}$} & \multirow{2}{*}{$\begin{array}{l}\text { M. sorbens abundance was greatest } \\
\text { summer and autumn. No statistical } \\
\text { measures. }\end{array}$} \\
\hline & & $\begin{array}{l}\text { Frequency: } 3 \text { x / month, June to } \\
\text { November (1998) and March to } \\
\text { November (1999), between } 8 \text { am to } \\
4 \text { pm }\end{array}$ & & \\
\hline $\begin{array}{l}\text { Location: Jinhua } \\
\text { City, Zhejiang } \\
\text { Province, } \\
\text { Southern China }\end{array}$ & & $\begin{array}{l}\text { Other details: traps baited with fish, } \\
\text { fermented bean curd and sugar. }\end{array}$ & $\begin{array}{l}\text { Mean monthly catches were highest } \\
\text { in October1998 and August } 1999 .\end{array}$ & \\
\hline \multirow{2}{*}{$\begin{array}{l}\text { Author(s): Liu at } \\
\text { al. (2010) [34] } \\
\text { Location: } \\
\text { Ankang City, } \\
\text { Shaanxi } \\
\text { Province, } \\
\text { Western China }\end{array}$} & \multirow[t]{2}{*}{$\begin{array}{l}\text { Adult } \\
\text { abundance }\end{array}$} & $\begin{array}{l}\text { Collection type: Traps (method not } \\
\text { reported) }\end{array}$ & \multirow{2}{*}{$\begin{array}{l}\text { Mean monthly catches appear to peak } \\
\text { from June to August, with the highest } \\
\text { peaks in July. }\end{array}$} & \multirow{2}{*}{$\begin{array}{l}\text { M. sorbens was the most populous } \\
\text { species caught accounting for } 48.7 \% \\
\text { of all flies caught. No statistical } \\
\text { measures. }\end{array}$} \\
\hline & & $\begin{array}{l}\text { Frequency: Time and duration of } \\
\text { catches unknown, January - } \\
\text { December } 2008 \text {. }\end{array}$ & & \\
\hline \multirow{3}{*}{$\begin{array}{l}\text { Author(s): He at } \\
\text { al. (2011) [31] } \\
\text { Location: } \\
\text { Qingyuan City, } \\
\text { Guangdong } \\
\text { Province, } \\
\text { Southern China }\end{array}$} & \multirow[t]{3}{*}{$\begin{array}{l}\text { Adult } \\
\text { abundance }\end{array}$} & Collection type: Conical traps & \multirow{3}{*}{$\begin{array}{l}\text { M. sorbens accounted for } 1.97 \% \text { of all } \\
\text { flies caught, but peaked in November } \\
\text { and January. }\end{array}$} & \multirow{3}{*}{$\begin{array}{l}\text { M. sorbens numbers appeared to peak } \\
\text { in November, and were lowest in } \\
\text { January. No statistical measures. }\end{array}$} \\
\hline & & $\begin{array}{l}\text { Frequency: } 1 \text { x / month, September } \\
2005 \text { - August } 2010\end{array}$ & & \\
\hline & & $\begin{array}{l}\text { Other details: Traps baited with } \\
\text { vinegar and sugar; deployed } 9 \text { am- } \\
12 \text { pm and 3-4 pm. }\end{array}$ & & \\
\hline
\end{tabular}

providing some possible insights related to seasonal effects on flies.

Three studies examined associations between distribution of flies and climatic factors [9, 22, 23].

\section{Temperature}

Toyama and Ikeda (1981) studied the role of climate factors on flies in a field study in Hawaii (Table 1) [23]. Summer season was significantly associated with greater abundance of all life stages of $M$. sorbens. The study also found that the crusts on dung pats at higher summer temperatures reduced predation, most likely by Labidura riparia, an earwig, which was unable to penetrate the pats to hunt fly larvae. In contrast, during the winter months, no such crust formed on the pats, and the earwigs depressed the population of $M$. sorbens.

\section{Altitude}

Taye et al. (2007) reported the abundance of $M$. sorbens caught on children's faces (indoors and outdoors) and in traps in Ethiopia, during four different seasons and at three altitudes, the latter being a proxy measure of ambient temperature (Table 1) [9]. Over $99 \%$ of M. sorbens were caught outdoors and only $0.7 \%$ of $M$ sorbens were caught on children living above $3,000 \mathrm{~m}$. At altitudes $<2000 \mathrm{~m}$ there was less seasonal variation in the median number of $M$. sorbens caught per child, ranging from 7/ child in the dry, winter season to 13/child in the wet, summer season. A different seasonal pattern and range was reported at higher altitude (2200-2,500 m) with the number of $M$. sorbens caught on children's faces ranging from $2.5 /$ child in the wet, summer season to $23 /$ child in the dry, winter season. Findings were similar from $M$. sorbens caught in beef-baited traps. At $<2000 \mathrm{~m}$ there 
were higher catches in all four seasons with not much seasonal variation (402 in the dry winter compared with 611 in semi-dry spring) compared with higher altitude (2200-2500 m) where the lowest counts were in the wet summer season $(11 \mathrm{M}$. sorbens) and highest in the semiwet autumn season ( $305 \mathrm{M}$. sorbens). The authors comment that at altitudes $<2000 \mathrm{~m}$ the annual temperature ranges from $23{ }^{\circ} \mathrm{C}$ in the wet season to $36{ }^{\circ} \mathrm{C}$ in the dry season and from 5 to $18{ }^{\circ} \mathrm{C}$ above $3,000 \mathrm{~m}$ but details were not published. There were no tests of association between season, altitude and $M$. sorbens counts.

\section{Relative humidity}

Hafez and Attia (1958) explored the impact of relative humidity and temperature on different aspects of $M$. sorbens life cycle (egg incubation period, egg hatching rate, duration of the larval and pupal periods, and egg structure (humidity only) in laboratory studies (Table 2) [22]. At $100 \%$ humidity, egg hatching occurred across a wide temperature range, with the optimal temperature for hatching being $28{ }^{\circ} \mathrm{C}$. At $70 \%$ humidity, adult emergence was maximal between 24 and $28{ }^{\circ} \mathrm{C}$, but did not occur above $40{ }^{\circ} \mathrm{C}$. When temperature was maintained at $31-32{ }^{\circ} \mathrm{C}$, eggs did not hatch at humidities below $80 \%$, but $100 \%$ of eggs hatched at $100 \%$ humidity (Fig. 5 and 6). None of these associations were assessed statistically.

The three higher quality papers demonstrate that, amongst the climatic factors studied, temperature appears to be the most strongly associated with $M$. sorbens abundance. Consistent with other Muscids, like $M$. domestica and $M$. autumnalis, $M$. sorbens have an optimum temperature for survival and behaviour, after which increasing temperatures are associated with a decrease in fly abundance [27-29]. Importantly, each species have different optimal conditions for their survival. The paper by Toyama et al. presents a plausible explanation why

\begin{tabular}{|c|c|c|c|c|c|}
\hline $\begin{array}{c}\text { Temperature } \\
\text { optimum ( }{ }^{\circ} \text { C) }\end{array}$ & Egg & Larva & Pupa & Adult & \\
\hline 42 & & & & & \\
40 & 1 & 3 & & & \\
38 & & & & & \\
36 & & & 4 & & \\
34 & 2 & & & & \\
32 & & & & & \\
30 & & & 5 & & \\
28 & & & & & \\
26 & & & & & \\
24 & & & & & \\
22 & & & & & \\
\hline
\end{tabular}

Fig. 5 Temperature Optima for Different Life Stages of M. sorbens, High and Moderate Quality Studies. 1. Shortest egg hatching time, Hafez \& Attia 1958 [21, 22]. 2. Highest percentage egg hatching, Hafez \& Attia 1958 [21, 22]. 3. Shortest larval period, Hafez \& Attia 1958 [21, 22]. 4. Shortest duration of pupal period, Hafez \& Attia 1958 [21, 22]. 5. Highest percentage of pupal hatching, Hafex \& Attia 1958 [21, 22]

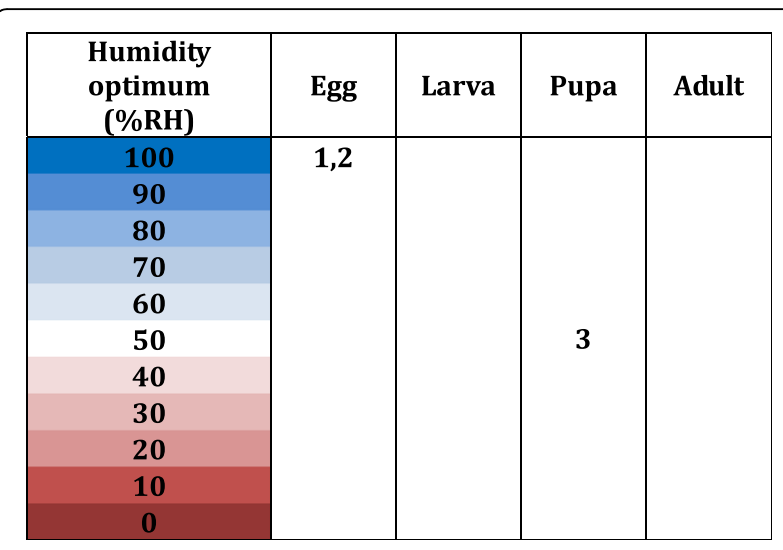

Fig. 6 Humidity Optima for Different Life Stages of M. sorbens, High and Moderate Quality Studies. 1. Shortest egg hatching time, Hafez \& Attia 1958 [21, 22]. 2. Highest percentage egg hatching, Hafez \& Attia 1958 [21, 22]. 3. Pupal phase duration was independent of humidity at humidities studied by Hafez \& Attia 1958 [21, 22]

M. sorbens no longer thrive at high temperatures (e.g., high temperature leading to crust formation on dung pats, thereby hindering fly emergence) [23].

The remaining 13 studies are described in Table 3 [21, 30-40]. These studies are mostly descriptive in nature, and report the relative abundance of $M$. sorbens in relation to other fly species, by season or month. Additional information on the climate for each location was obtained to see if there was consistency across the climate factors that described the peak fly season.

\section{Discussion and conclusion}

This review of potential associations between climatic factors and $M$. sorbens was conducted in order to provide evidence on the climatic boundaries beyond which $M$. sorbens is unlikely to be a vector of trachoma. Outside these climatic constraints, transmission of $C$. trachomatis is more likely to be through person-to-person contact rather than via flies. Consequently, in such conditions, interventions should focus on facial cleanliness, other behaviours, and reductions in overcrowding. It was envisioned that insights gained could also assist in planning future trachoma control strategies in the face of possible global climate change. However, this systematic literature review indicates that evidence for the effect of climate factors on the abundance and seasonal activity of $M$. sorbens is very limited.

One reason for the lack of evidence regarding climatic factors which affect $M$. sorbens is that the fly has only recently been implicated as an important route of transmission in trachoma [7]. In contrast, the vectors of many other infectious diseases (e.g., malaria, dengue, leishmaniasis and trypanosomiasis) have been studied for years in order to improve vector control methods. Maps of disease vectors generally lag behind those of the diseases themselves, although the recently-produced Malaria Atlas provides 
a comprehensive, well-funded project mapping the distribution of the forty one dominant malaria vector species [41]. The effect of climatic factors on the biology of malaria vectors has also been studied, allowing for the development of models that forecast possible effects of climate change on the distribution of malaria vectors and hence vectorial capacity [42, 43]. Whilst the Trachoma Atlas is currently being compiled to map the distribution and endemicity of trachoma, at present there is no equivalent map of the distribution of $M$. sorbens and the effects of climatic factors on the abundance and distribution of this fly remain poorly understood in comparison to the state of knowledge on malaria vectors [3].

Although there is still some uncertainty about the relative importance of transmission by $M$. sorbens, results of this review support the implementation of the SAFE ( $\mathrm{S}$ = surgery, $\mathrm{A}=$ mass antibiotic distribution, $\mathrm{F}=$ facial cleanliness to reduce ocular secretions and, $\mathrm{E}=$ environmental hygiene) strategy, which is being led and scaled-up by the WHO Alliance for the Global Elimination of Blinding Trachoma (GET 2020) with the aim of eliminating blinding trachoma by the year 2020 [32]. In particular, the aim of the ' $E$ ' component of the strategy is to reduce $C$. trachomatis transmission by means of environmental improvements such as water and sanitation; increased access to water could improve hygiene, while reductions in open defecation - which provides ample, open breeding sites for $M$. sorbens could result in reductions in trachoma [6, 44].

This systematic review had several limitations. First, given the dearth of moderate and high quality evidence related to the effects of climate factors on $M$. sorbens, it was difficult to interpret the results of the very limited evidence base. The sixteen studies included in this review employed a wide variety of designs, methods, and analytical tools. Only one study provided evidence from a laboratory setting, and this study was conducted nearly 60 years ago, when methods and analytical techniques were less advanced than at present [22]. The highest quality papers were both conducted within the last 30 years and used statistical methods to provide evidence of the strength of associations [9, 23]. Unfortunately most studies reviewed did not provide statistical evidence. None of the studies used robust designs, and none provided details on all of the entomological methods used (e.g., trap designs, speciation methods, etc.). These limitations mean that most studies were categorized as being of poor quality and the results were difficult to interpret.

There is a need for more evidence on the relationship between climatic factors and $M$. sorbens distribution and abundance which could be used to guide whether the $\mathrm{F}$ and/or E elements of the SAFE strategy need to be prioritized. For example, interventions in locations where climatic conditions promote $M$. sorbens abundance all the year round need to focus on all elements of the SAFE strategy. In locations where climatic factors promote seasonal variation in the abundance of $M$ sorbens targeted vector control could be considered. In locations where climatic factors are not conducive to $M$. sorbens the $\mathrm{F}$ elements need to be promoted to reduce transmission via fomites.

This review found a dearth of data linking climate variables with important life-history traits of $M$. sorbens. Such data are typically used to construct mathematical models that allow investigators to explore the impact of climate change on the risk of vector-borne diseases. One alternative approach that is used commonly by biologists where detailed data on a species is lacking is to produce maps based on the climate at sites where a species was recorded i.e., presence only data [45]. Machine-learning techniques and community models, that are well suited to sparse occurrence data, are then used to map a species' distribution based on climate factors. The statistical relationship that best captures the climate envelope for the fly can be used to explore future distributions when it is linked to future climate change scenarios [46]. Such an approach would require collating data on the distribution of this species from museum collections, record lists and active surveys.

An alternative approach is to build mathematical models that describe the relationship between the life stages of $M$. sorbens and climate based on what is known for other Muscids. For example the relationship between climate and the bionomics of the house fly, $M$. domestica, and the face fly, M. autumnalis, have been described in more detail [27-29]. These models could be used to help produce models for $\mathrm{M}$. sorbens where there are few data.

Overall, M. sorbens is adapted to hot tropical climates and can withstand highly desiccating environments. It breeds most actively in hot humid conditions, but this relationship is likely to be non-linear with extremely high temperatures reducing fly survival. Indirect effects of climate on fly abundance are difficult to predict, but higher temperatures and low humidity may create a hard crust on stools harbouring immature flies, restricting the emergence of the young flies and preventing predation of the immature stages. During the wet season dung beetles may remove surface faeces so quickly that flies may have to compete for a severely limited resource resulting in higher numbers of flies per stool with density-dependent effects coming into play and further reducing the number of flies emerging. Perhaps most importantly we are uncertain whether flies carry Chlamydia on the surface of the fly or internally. If the pathogen is found largely on the body of a fly it will be 
more vulnerable to the effects of desiccation, whilst if carried internally it will be most sensitive to changes in temperature. Whilst many of these suggestions are conjectures, they do illustrate the potential complexity of the system and how difficult it is to predict how climate factors influence the risk of trachoma transmission.

More data on how climatic variables influence the current abundance of $M$. sorbens will allow forecasts to be made concerning the potential impact of future climate change, indicating areas where the role of $M$. sorbens as a vector of trachoma may vary in importance over time.

\section{Additional file}

Additional file 1: Table S1. Search Terms for Systematic Literature Review. (DOCX $13 \mathrm{~kb}$ )

\section{Competing interests}

The authors declare that they have no competing interests.

\section{Authors' contributions}

$A R$ and JB conducted the systematic literature review as first and second reader, respectively. $A R, J B, S K, S L$, and $C G$ analysed the findings. $A R$ wrote the first draft of the manuscript. AR, JB, SK, SL, CG, DH, and ES contributed to the study design and writing of the manuscript. All authors read and approved the final version of the manuscript.

\section{Acknowledgements}

$A R, J B$, and SK were supported by Sightsavers.

\section{Author details}

${ }^{1}$ Department of Clinical Research, Faculty of Infectious and Tropical Diseases (ITD), London School of Hygiene \& Tropical Medicine (LSHTM), Keppel Street, London, UK. ²Department of Disease Control, ITD, LSHTM, Keppel Street, London, UK. ${ }^{3}$ Department of Social and Environmental Health Research, Faculty of Public Health and Policy, LSHTM, Keppel Street, London, UK. ${ }^{4}$ School of Biological and Biomedical Sciences, Durham University, Durham, UK. ${ }^{5}$ Sightsavers, Haywards Health, West Sussex, UK.

Received: 13 November 2015 Accepted: 20 January 2016 Published online: 27 January 2016

\section{References}

1. World Health Organization (WHO). Trachoma Geneva: WHO; 2015 [cited 2015 July 25 2015]. Available from: http://www.who.int/mediacentre/ factsheets/fs382/en/

2. International Coalition for Trachoma Control (ICTC). The International Coalition for Trachoma Control (ICTC) 2015 [cited 2015 July 25, 2015]. Available from: http://www.trachomacoalition.org/.

3. Smith JL, Haddad D, Polack S, Harding-Esch EM, Hooper PJ, Mabey DC, et al. Mapping the global distribution of trachoma: why an updated atlas is needed. PLoS Negl Trop Dis. 2011:5(6):e973.

4. World Health Organization (WHO). Trachoma: Prevention of Blindness and Visual Impairment 2015 [cited 2015 July 25, 2015]. Available from: http://www.who.int/blindness/causes/trachoma/en/.

5. Emerson PM, Bailey RL, Mahdi OS, Walraven GE, Lindsay SW. Transmission ecology of the fly Musca sorbens, a putative vector of trachoma. Trans R Soc Trop Med Hyg. 2000;94(1):28-32.

6. Emerson PM, Bailey RL, Walraven GE, Lindsay SW. Human and other faeces as breeding media of the trachoma vector Musca sorbens. Med Vet Entomol. 2001;15(3):314-20.

7. Emerson PM, Lindsay SW, Alexander N, Bah M, Dibba SM, Faal HB, et al. Role of flies and provision of latrines in trachoma control: cluster-randomised controlled trial. Lancet. 2004;363(9415):1093-8.
8. Emerson PM, Simms VM, Makalo P, Bailey RL. Household pit latrines as a potential source of the fly Musca sorbens-a one year longitudinal study from The Gambia. Trop Med Int Health. 2005;10(7):706-9.

9. Taye A, Alemayehu W, Melese M, Geyid A, Mekonnen Y, Tilahun D, et al. Seasonal and altitudinal variations in fly density and their association with the occurrence of trachoma, in the Gurage zone of central Ethiopia. Ann Trop Med Parasitol. 2007;101(5):441-8.

10. Johnson GJ. The environment and the eye. Eye (Lond). 2004;18(12):1235-50.

11. West SK, Emerson P, Mkocha H, McHiwa W, Munoz B, Bailey R, et al. Intensive insecticide spraying for fly control after mass antibiotic treatment for trachoma in a hyperendemic setting: a randomised trial. Lancet. 2006;368(9535):596-600.

12. Service MW. Medical Entomology for Students. 5th Edition ed. Cambridge: Cambridge University Press; 2014

13. Ramesh A, Kovats S, Haslam D, Schmidt E, Gilbert CE. The impact of climatic risk factors on the prevalence, distribution, and severity of acute and chronic trachoma. PLoS Negl Trop Dis. 2013;7(11):e2513.

14. Afrane YA, Githeko AK, Yan G. The ecology of Anopheles mosquitoes under climate change: case studies from the effects of deforestation in East African highlands. Ann N Y Acad Sci. 2012;1249:204-10.

15. Andrew NR, Hill SJ, Binns M, Bahar MH, Ridley EV, Jung MP, et al. Assessing insect responses to climate change: What are we testing for? Where should we be heading? PeerJ. 2013;1:e11.

16. Root TL, Price JT, Hall KR, Schneider SH, Rosenzweig C, Pounds JA. Fingerprints of global warming on wild animals and plants. Nature. 2003; 421(6918):57-60.

17. Dakshinamurty S. The common house-fly, Musca domestica, and its behaviour to temperature and humidity. Bull Entomol Res. 1948;39(Pt. 3):339-57.

18. Folk DG, Han C, Bradley TJ. Water acquisition and partitioning in Drosophila melanogaster: effects of selection for desiccation-resistance. J Exp Biol. 2001;204(Pt 19):3323-31.

19. Hoffmann AA, Hallas R, Sinclair C, Mitrovski P. Levels of variation in stress resistance in drosophila among strains, local populations, and geographic regions: patterns for desiccation, starvation, cold resistance, and associated traits. Evolution. 2001;55(8):1621-30.

20. Ngoen-klan R, Moophayak K, Klong-klaew T, Irvine KN, Sukontason KL, Prangkio C, et al. Do climatic and physical factors affect populations of the blow fly Chrysomya megacephala and house fly Musca domestica? Parasitol Res. 2011;109(5):1279-92.

21. Hafez M, Attia MA. On the developmental stages of Musca sorbens Wied. with special reference to larval behaviour (Diptera: Musoidae). Bull Soc ent Egypte. 1958;42:123-61.

22. Hafez M, Attia MA. Studies on the ecology of Musca sorbens Wied. in Egypt (Diptera: Muscidae). Bull Soc ent Egypte. 1958;42:83-121.

23. Toyama GM, Ikeda JK. Predation as a factor in seasonal abundance of Musca sorbens Wiedemann (Diptera: Muscidae). Proc Hawaiian Entomol Soc. 1981; 23(3):447-54.

24. Hanski ICY. Dung Beetle Ecology: Princeton Legacy Library. 1991. p. 520

25. Mbilu TJNK, Silayo RS, Kimbita EN, Onditi SJ. Studies on the importance of the face Fly Musca sorbens at Kambala Village, Mvomero District, Morogoro, Tanzania. Livestock Res Rural Dev. 2007; 19(46). Retrieved September 1, 2015, from http://www.Irrd.org/Irrd19/ 4/mbil19046.htm.

26. National Collaborating Centre for Methods and Tools. Quality Assessment Tool for Quantitative Studies. Hamilton, ON: McMaster University; 2008. (Updated 13 April, 2010) Retrieved from: http://www.nccmt.ca/resources/ search/14.

27. Teskey HJ. On behavior and ecology of the face fly, Musca autumnalis (Diptera-Muscidae). Can Entomol. 1969;101:561-76.

28. Valiela I. An experimental study of the mortality factors of larval Musca autumnalis DeGeer. Ecology Monogr. 1969;39:199-225.

29. West LR. The Housefly. New York: Comstock Publishing; 1951

30. Amin ARH, Morsy TA, Shoukry A, Mazyad SAM. Studies on myiasis producing flies collected by bait traps at Al Marg (Qalyobia Governorate), Egypt. J Egypt Soc Parasitol. 1998;28(1):45-51.

31. He Z, Wang G, Li C, Zhu X, He Y, Zhou K, et al. Background survey and fluctuation research of flies population in Qingyuan urban area. Occup Health. 2011;27(21):2482-4.

32. Khan NH, Ansari JA, Rehman J, Ahmad D. Relative abundance of houseflies in India and their susceptibility to DDT, BHC, and dieldrin. J Bombay Nat Hist Soc. 1965;61(3):712-6. 
33. Koe FH. Preliminary observations on the species constitution and the seasonal fluctuations of synanthropic flies in the plain rural region of Honan Province. Acta Entomologica Sinica. 1975;18(1):71-6.

34. Liu P, Zhou Y, Wang Z, Yuan L, Ma X, Zhang Y. The investigation of the seasonal fluctuation of vectors in Ankang city. Chin J Vector Biol Control. 2010;21(6):596-8.

35. Miranpuri GS, Lahkar BC. Seasonal incidence of dipterous flies associated with cattle at Gauhati, Assam. Indian J Parasitol. 1980;4(2):183-5.

36. Ponghis $\mathrm{G}$. Some observations on the part of the house fly in the transmission of seasonal conjunctivitis in Southern Morocco. [French] Quelques observations sur le role de la mouche dans la transmission des conjonctivites saisonnieres dans le Sud-Marocain. Bull World Health Organ. 1957;16(5):1013-27.

37. Rechav Y. Seasonal abundance of Muscidae (Diptera) associated with cattle on a northern Transvaal farm. J Med Entomol. 1989:26(4):368-9.

38. Sukhova MN. Control measures against Musca sorbens in the prevention of acute epidemic conjunctivitis. J Hyg Epidemiol Microbiol Immunol. 1963; 7(1):82-9.

39. Tawfik MS. Ecological studies on some desert flies in Egypt. Indian J Entomol. 1969;31(3):201-21.

40. Wang $F$, Bao $Y$, Wang $H$. Season variety of fly density in Jinhua city area. Chin J Vector Biol Control. 2000;11(4):279-81.

41. Sinka ME, Bangs MJ, Manguin S, Rubio-Palis Y, Chareonviriyaphap T, Coetzee M, et al. A global map of dominant malaria vectors. Parasit Vectors. 2012;5:69.

42. Lindsay SW, Bayoh MN. Mapping members of the Anopheles gambiae complex using climate data. Physiol Entomol. 2004;29:204-9.

43. Tonnang HE, Kangalawe RY, Yanda PZ. Predicting and mapping malaria under climate change scenarios: the potential redistribution of malaria vectors in Africa. Malar J. 2010;9:111.

44. Lindsay SW, Lindsay TC, Duprez J, Hall MJ, Kwambana BA, Jawara M, et al. Chrysomya putoria, a putative vector of diarrheal diseases. PLoS Negl Trop Dis. 2012;6(11):e1895.

45. Elith J, Graham CH, Anderson RP, Dudík M, Ferrier S, Guisan A, et al. Novel methods improve prediction of species' distributions from occurrence data. Ecography. 2006;29:129-51.

46. Pearson RG, Dawson TP. Predicting the impacts of climate change on the distribution of species: are bioclimate envelope models useful? Glob Ecol Biogeogr. 2003;12:361-71.

\section{Submit your next manuscript to BioMed Central and we will help you at every step:}

- We accept pre-submission inquiries

- Our selector tool helps you to find the most relevant journal

- We provide round the clock customer support

- Convenient online submission

- Thorough peer review

- Inclusion in PubMed and all major indexing services

- Maximum visibility for your research

Submit your manuscript at www.biomedcentral.com/submit 